MATHEMATICS OF COMPUTATION

Volume 68, Number 225, January 1999, Pages 385-388

S 0025-5718(99)01048-0

\title{
ON TWO CLASSES OF SIMULTANEOUS PELL EQUATIONS WITH NO SOLUTIONS
}

\author{
P. G. WALSH
}

\begin{abstract}
In this paper we describe two classes of simultaneous Pell equations of the form $x^{2}-d y^{2}=z^{2}-e y^{2}=1$ with no solutions in positive integers $x, y, z$. The proof is elementary and covers the case $(d, e)=(8,5)$, which was solved by E. Brown using very deep methods.
\end{abstract}

\section{INTRODUCTION}

There have been several papers written that deal with the simultaneous solution to two Pell equations. The general form of this system of equations is

$$
\begin{aligned}
& a x^{2}-b y^{2}=c, \\
& d z^{2}-e y^{2}=f,
\end{aligned}
$$

where $a, b, c, d, e, f$ are nonzero integers such that the two equations are not multiples of one another. It follows from the work of Thue [12] and Siegel [10] that these systems have only a finite number of solutions in integers $x, y, z$. Furthermore, Baker's theorem on linear forms in logarithms of algebraic numbers [1] provides a method to compute an upper bound for the size of the solutions. In [2], Baker and Davenport used such an upper bound along with techniques from diophantine approximation and lengthy multiprecise computations to prove that the system $x^{2}-3 y^{2}=-2, z^{2}-8 y^{2}=-7$ has only the solutions $(x, y, z)=(1,1,1),(19,11,31)$. Grinstead [7] used Baker's theorem but refined the technique of Baker and Davenporte $([2])$ to show that the system $x^{2}-8 y^{2}=1,3 z^{2}-2 y^{2}=1$ has no solutions. Brown [4] used Grinstead's method, together with Baker's theorem to show that the only solution to the system $x^{2}-8 y^{2}=1, z^{2}-5 y^{2}=1$ is $(x, y, z)=(1,0,1)$.

The purpose of this paper is to use elementary methods to describe two classes of simultaneous Pell equations which have no nontrivial solutions. In particular, one of the two classes contains the system considered by Brown in [4]. Thus, Theorem 1 generalizes the result in [4], and moreover, the proof relies only on basic properties of solutions to Pell's equation and the nonexistence of squares in a certain second order linear recurrence.

Theorem 1. Let $a \geq 1$ be an integer. Let $q$ be a prime such that $x^{2}-2 q y^{2}=-1$ is solvable, and such that $\left|2^{2 a+1}-q\right|=p^{b}$ for some prime $p$ and $b \geq 0$. Then the

Received by the editor February 2, 1996

1991 Mathematics Subject Classification. Primary 11D09.

Supported by an N.S.E.R.C. Posdoctoral Fellowship. 
system of simultaneous Pell equations

$$
\begin{gathered}
x^{2}-2^{2 a+1} y^{2}=1, \\
z^{2}-q y^{2}=1
\end{gathered}
$$

has only the trivial solution $(x, y, z)=(1,0,1)$.

If $a=1$ and $q=5$, then the hypotheses of Theroem 1 are satisfied, and this is the system considered by Brown in [4]. We remark that by the main result of Trotter in [11], $x^{2}-2 q y^{2}=-1$ is solvable for $q \equiv 5(\bmod 8)$, and so this hypothesis can be removed from Theorem 1 for these primes.

Theorem 2. Let $d=2^{2 a} c$ and $e$ be integers, with $c>1$ odd, $a \geq 1$, and $e$ odd, such that de $=2^{2 a} k^{4} D$ for positive integers $k, D$ with the property that the Pell equation $x^{2}-D y^{2}=-4$ has solutions in odd integers $x, y$. If $|d-e|=p^{b}$ for some prime $p$ and $b \geq 0$, then the system of simultaneous Pell equations

$$
\begin{gathered}
x^{2}-d y^{2}=1, \\
z^{2}-e y^{2}=1
\end{gathered}
$$

has only the trivial solution $(x, y, z)=(1,0,1)$.

This result shows for example that no nontrivial solutions exist to (3) and (4) when $d=20$ and $e=17$. Using the results of Cohn ([6]), one may extend this result to those $D$ for which $x^{2}-D y^{2}=-4$ has no odd solutions $x$ and $y$, but for which $x^{2}-D y^{2}=4$ has odd solutions. We forgo this analysis. The main ingredient in the proof of this result is the absence of squares in the set of values of $y$ of solutions to $x^{2}-D y^{2}=1$, proved by Cohn in [5] and [6]. An interesting open problem, which would have application to extending Theorem 2, is to prove an analogous result of Cohn's for those values of $D$ for which $x^{2}-D y^{2}=4$ has no solutions in odd integers $x$ and $y$. Bennett [3] has recently proved that the system (3) and (4) has at most 3 solutions for arbitrary but distinct positive integers $d$ and $e$. His method uses the theory of linear forms in the logarithms of algebraic numbers.

\section{Proofs}

The proofs of Theorems 1 and 2 rely on properties of solutions to Pell equations. The reader is referred to [8] for a description of these properties.

Proof of Theorem 1. We will assume that $2^{2 a+1}>q$, as the other case is proved in the same manner. Put $t=p^{b}$, then subtracting (2) from (1) yields $x^{2}-z^{2}=t y^{2}$. From the hypotheses it follows that $q \equiv 1(\bmod 4)$, and hence from $(1)$ and $(2)$ we see that both $x$ and $z$ are odd, and that $\operatorname{gcd}(x-z, x+z)=2$. Since $t$ is a power of an odd prime, it follows that there are positive integers $A, B$, with $y=2 A B$, such that either $x-z=2 t A^{2}, x+z=2 B^{2}$ or $x-z=2 B^{2}, x+z=2 t A^{2}$. In either case we deduce that $x=B^{2}+t A^{2}$. Substituting this into (1) yields

$$
B^{4}+\left(2 t-4 \cdot 2^{2 a+1}\right) B^{2} A^{2}+t^{2} A^{4}-1=0,
$$

which is the same as

$$
\left(B^{2}-\left(2^{2 a+1}+q\right) A^{2}\right)^{2}-2^{2 a-1} q(2 A)^{4}=1 .
$$

Let $T+U \sqrt{2 q}$ denote the fundamental solution to the Pell equation $x^{2}-2 q y^{2}=-1$, and for $i \geq 1$ let $T_{i}+U_{i} \sqrt{2 q}=(T+U \sqrt{2 q})^{i}$. From the divisibility properties of 
solutions to $x^{2}-2 q y^{2}= \pm 1$ (see [8]), 4 divides $U_{i}$ if and only if 4 divides $i$. From (5) we find that there must be an integer $i$ such that

$$
\left|B^{2}-\left(2^{2 a+1}+q\right) A^{2}\right|+\left(2^{a+1} A^{2}\right) \sqrt{2 q}=T_{4 i}+U_{4 i} \sqrt{2 q} .
$$

Again from the properties of solutions to Pell equations we have that $U_{4 i}=2 T_{2 i} U_{2 i}$, $\operatorname{gcd}\left(T_{2 i}, U_{2 i}\right)=1, T_{2 i}$ is odd, and $U_{2 i}$ is even. It follows that there are positive integers $C$ and $E$ such that $T_{2 i}=C^{2}$ and $U_{2 i}=2^{a} E^{2}$. Thus there is a positive integer $F$ such that either

$$
C^{4}-1=2 q F^{4}
$$

or

$$
C^{4}-1=8 q F^{4}
$$

depending on whether $a$ is even or odd. Since $C$ is odd, $\operatorname{gcd}\left(C^{2}+1, C^{2}-1\right)=2$, and 2 properly divides $C^{2}+1$. It follows from either of (6) and (7) that there is a positive integer $G$ such that either $C^{2}+1=2 G^{4}$ or $C^{2}+1=2 q G^{4}$.

By the result of [9], the first of these two possibilities implies that either $G=1$ or $G=13$. If $G=13$, then $C=239$, and it is easily checked that $C^{4}-1$ is not of the form $8 q F^{4}$ or $2 q F^{4}$. Therefore this case does not lead to a solution of (1) and (2). The case $G=1$ results in the trivial solution $(x, y, z)=(1,0,1)$.

The second possibility implies that there is an odd index $l$ such that

$$
C+G^{2} \sqrt{2 q}=T_{l}+U_{l} \sqrt{2 q}
$$

We show that this cannot occur. Let $P$ denote a prime dividing $C^{2}=T_{2 i}$. Then since $\operatorname{gcd}\left(T_{2 i}, U_{2 i}\right)=1, P$ divides $U_{4 i}=2 T_{2 i} U_{2 i}$, but $P$ does not divide $U_{2 i}$. By the divisibility properties of solutions to Pell's equation, it follows that 4 divides any index $j$ for which $P$ divides $U_{j}$. But $P$ divides $T_{l}$, and hence $P$ divides $U_{2 l}=2 T_{l} U_{l}$, which is not possible from the fact that $l$ is odd.

Proof of Theorem 2. We will assume that $d>e$, as the other case is proved in the same manner. Let $t=d-e$, then subtracting (4) from (3) yields $x^{2}-z^{2}=t y^{2}$. It is evident that $\operatorname{gcd}(x-z, x+z)=1$ or 2 . Thus, either $x \pm z=t A^{2}, x \mp z=B^{2}$ with $y=A B$, or $x \pm z=2 t A^{2}, x \mp z=2 B^{2}$ with $y=2 A B$. We deduce that either $2 x=t A^{2}+B^{2}$ in the first case, or $x=t A^{2}+B^{2}$ in the second case. Substituting these into (3), simplifying, and using the quadratic formula, we find that

$$
B^{2}=(d+e) \pm 2 \sqrt{d e A^{4}+1}
$$

in the first case, and

$$
B^{2}=(d+e) \pm \sqrt{4 d e A^{4}+1}
$$

in the second case. From (8) there is an integer $X$ such that

$$
X^{2}-\operatorname{de} A^{4}=1
$$

and from (9) there is an integer $X$ such that

$$
X^{2}-4 d e A^{4}=1 \text {. }
$$

From (10) we have that $X^{2}-2^{2 a}(k A)^{4} D=1$. If $a$ is odd we appeal to [5, p. 163 , Equation 3] to find that $D=5,2^{(a-1) / 2} k A=6$, and $X=161$. For all possible choices of $d$ and $e$ satisfying the hypotheses of the theorem we find that the right hand side of (8) is not a square. If $a$ is even we appeal to [5, p. 163, Equation $1]$ to find that $D=5$ and $2^{a / 2} k A=2$. In this case there are no possible values 
of $d$ and $e$ satisfying the hypotheses of the Theorem 2. From (11) we have that $X^{2}-2^{2 a+2}(k A)^{4} D=1$. In the same manner as above we deduce from [5] that there are no possible values of $d$ and $e$ which yield nontrivial solutions to (3) and (4).

\section{REFERENCES}

[1] A. BAKER. Linear Forms in the logarithms of algebraic numbers. Mathematika 15 (1968), 204-216. MR 41:3402

[2] A. Baker And H. Davenport. The equations $3 x^{2}-2=y^{2}$ and $8 x^{2}-7=z^{2}$. Quart. J. Math. Oxford (2) 20 (1969), 129-137. MR 40:1333

[3] M.A. BennetT. On the number of solutions to simultaneous Pell equations. To appear in J. Reine. Angew. Math.

[4] E. Brown. Sets in which $x y+k$ is always a square. Math. Comp. 45 (1985), 613-620. MR 86k:11019

[5] J.H.E. Cohn. Eight Diophantine equations. Proc. London Math. Soc. (3) 16 (1966), 153-166. MR 32:7492

[6] J.H.E. Cohn. Five Diophantine equations. Math. Scand. 21 (1967), 61-70. MR 38:4401

[7] C.M. Grinstead. On a method of solving a class of Diophantine equations. Math. Comp. 32 (1978), 936-940. MR 58:10724

[8] D.H. Lehmer. An extended theory of Lucas functions. Ann. Math. 31 (1930), 419-448.

[9] W. Lunnggren. Zur Theorie der Gleichung $x^{2}+1=D y^{4}$. Avh. Norsk. Vid. Akad. Oslo (1942), 1-27. MR 8:6f

[10] C.L. Siegel. Uber einige Anwendungen diophantischer Approximationen. Abh. Preuss. Akad. Wiss. (1929), 1.

[11] H.F. Trotter. On the norm of units in quadratic fields. Proc. Amer. Math. Soc. 22 (1969), 198-201. MR 39:5513

[12] A. Thue. Uber Annaherungenswerte algebraischen Zahlen. J. Reine Angew. Math. 135 (1909), 284-305.

Department of Mathematics, University of Ottawa, 585 King Edward Street, OtTAWA, Ontario, K1N-6N5 Canada

E-mail address: gwalsh@mathstat.uottawa.ca 